\title{
ON CONJUGACY OF HIGH-ORDER LINEAR ORDINARY DIFFERENTIAL EQUATIONS
}

\author{
T. CHANTURIA $†$
}

ABStract. It is shown that the differential equation

$$
u^{(n)}=p(t) u,
$$

where $n \geq 2$ and $p:[a, b] \rightarrow \mathbb{R}$ is a summable function, is not conjugate in the segment $[a, b]$, if for some $l \in\{1, \ldots, n-1\}, \quad \alpha \in] a, b[$ and $\beta \in] \alpha, b[$ the inequalities

$$
\begin{gathered}
n \geq 2+\frac{1}{2}\left(1+(-1)^{n-l}\right), \quad(-1)^{n-l} p(t) \geq 0 \text { for } t \in[a, b], \\
\int_{\alpha}^{\beta}(t-a)^{n-2}(b-t)^{n-2}|p(t)| d t \geq l !(n-l) ! \frac{(b-a)^{n-1}}{(b-\beta)(\alpha-a)},
\end{gathered}
$$

hold.

Consider the differential equation

$$
u^{(n)}=p(t) u,
$$

where $n \geq 2, p \in L_{l o c}(I)$, and $I \subset \mathbb{R}$ is an interval.

The following definitions will be used below.

Equation (1) is said to be conjugate in $I$ if there exists a nontrivial solution of this equation with at least $n$ zeroes (each zero counted accordingly to its multiplicity) in $I$.

Let $l \in\{1, \ldots, n-1\}$. Equation (1) is said to be $(l, n-l)$ conjugate in $I$ if there exists a nontrivial solution $u$ of this equation satisfying

$$
\begin{array}{ll}
u^{(i)}\left(t_{1}\right)=0 & (i=0, \ldots, l-1), \\
u^{(i)}\left(t_{2}\right)=0 & (i=0, \ldots, n-l-1),
\end{array}
$$

with $t_{1}, t_{2} \in I$ and $t_{1}<t_{2}$.

Suppose first that $-\infty<a<b<+\infty$ and $p \in L([a, b])$.

1991 Mathematics Subject Classification. 34C10. 
Lemma. Let $a<\alpha<\beta<b$. Then the Green's function $G$ of the problem

$$
\begin{array}{ll}
u^{(n)}(t)=0 & \text { for } t \in[a, b], \\
u^{(j)}(a)=0 & (j=0, \ldots, l-1), \\
u^{(j)}(b)=0 & (j=0, \ldots, n-l-1),
\end{array}
$$

satisfies the inequality

$$
\begin{aligned}
& (-1)^{n-l} G(t, s)> \\
& >\frac{(b-\beta)(\alpha-a)(s-a)^{n-l-1}(b-s)^{l-1}(t-a)^{l-1}(b-t)^{n-l-1}}{(b-a)^{n-1}} \times \\
& \times \sum_{i=1}^{n-l} \frac{(-1)^{n-l-i}}{(i-1) !(n-i) !} \quad \text { for } \alpha \leq t<s \leq \beta .
\end{aligned}
$$

Proof. The function $G$ can be written in the form

$$
G(t, s)=\left\{\begin{array}{ccc}
\sum_{i=n-l+1}^{n}(-1)^{i-1} x_{i}(t) x_{n-i+1}(s) & \text { for } & a \leq s<t \leq b, \\
-\sum_{i=1}^{n-l}(-1)^{i-1} x_{i}(t) x_{n-i+1}(s) & \text { for } & a \leq t \leq s \leq b,
\end{array}\right.
$$

where

$$
x_{i}(t)=\frac{(t-a)^{n-i}(b-t)^{i-1}}{(i-1) !(b-a)^{n-i}} .
$$

It is easy to verify that for any fixed $s \in] a, b\left[\right.$ the function $\frac{(-1)^{n-l} G(\cdot, s)}{x_{n-l}(\cdot) x_{l+1}(s)}$ decreases on $] a, b\left[\right.$ and the function $\frac{(-1)^{n-l} G(\cdot, s)}{x_{n-l+1}(\cdot) x_{l}(s)}$ increases on $] a, b[$. Thus

$$
(-1)^{n-l} G(t, s) \geq(-1)^{n-l} G(s, s) \frac{x_{n-l}(t)}{x_{n-l}(s)} \text { for } t \leq s .
$$

Taking into account that

$$
\begin{aligned}
(-1)^{n-l} G(s, s) & =(-1)^{n-l-1} \sum_{i=1}^{n-l}(-1)^{i-1} x_{i}(s) x_{n-i+1}(s)= \\
& =\frac{(s-a)^{n-1}(b-s)^{n-1}}{(b-a)^{n-1}} \sum_{i=1}^{n} \frac{(-1)^{n-l-i}}{(i-1) !(n-i) !}
\end{aligned}
$$

and

$$
\frac{x_{n-l}(t)}{x_{n-l}(s)}=\frac{(t-a)^{l}(b-t)^{n-l-1}}{(s-a)^{l}(b-s)^{n-l-1}}
$$


from the inequality (3) we deduce

$$
\begin{aligned}
& (-1)^{n-l} G(t, s) \geq \\
& \geq \frac{(s-a)^{n-l-1}(b-s)^{l}(t-a)^{l}(b-t)^{n-l-1}}{(b-a)^{n-1}} \sum_{i=1}^{n-l} \frac{(-1)^{n-l-i}}{(i-1) !(n-i) !}> \\
& >(b-\beta)(\alpha-a) \frac{(s-a)^{n-l-1}(b-s)^{l-1}(t-a)^{l-1}(b-t)^{n-l-1}}{(b-a)^{n-1}} \times \\
& \times \sum_{i=1}^{n-l} \frac{(-1)^{n-l-i}}{(i-1) !(n-i) !} \text { for } \quad \alpha \leq t<s \leq \beta .
\end{aligned}
$$

Theorem 1. Let $l \in\{1, \ldots, n-1\}$,

$$
n \geq 2+\frac{1+(-1)^{n-l}}{2} \text { and }(-1)^{n-l} p(t) \geq 0 \text { for } t \in[a, b] .
$$

If, in addition, there exist $\alpha, \beta \in] a, b[$ such that $a<\alpha<\beta<b$ and

$$
\int_{\alpha}^{\beta}(t-a)^{n-2}(b-t)^{n-2}|p(t)| d t \geq l !(n-l) ! \frac{(b-a)^{n-1}}{(b-\beta)(\alpha-a)},
$$

then Eq. (1) is $(l, n-l)$ conjugate in $[a, b]$.

Note that analogous results are given in $[3,5]$ for the case where $n=2$.

Proof. Put $p(t)=0$ for $t>b$ and consider Eq. (1) in the interval $[a,+\infty[$. For any $\gamma>a$, let $u_{\gamma}$ be the solution of (1) satisfying

$$
\begin{aligned}
& u_{\gamma}^{(i)}(a)=0 \quad(i=0, \ldots, l-1), \\
& u_{\gamma}^{(i)}(\gamma)=0 \quad(i=0, \ldots, n-l-2), \\
& \sum_{i=0}^{n-1}\left|u_{\gamma}^{(i)}(a)\right|=1, \quad \max \left\{u_{\gamma}(t): a \leq t \leq \gamma\right\}>0 .
\end{aligned}
$$

Suppose now that in spite of the statement of the theorem Eq. (1) is not $(l, n-l)$ conjugate in $[a, b]$.

Note that if $\gamma \in] a, b]$, then $u_{\gamma}(t)>0$ for $\left.t \in\right] a, \gamma\left[\right.$ and $(-1)^{n-l-1} \times$ $\times u_{\gamma}^{(n-l-1)}(\gamma)>0$. Indeed, if it is not so, there exists $\left.t_{0} \in\right] a, \gamma[$ such that $u_{\gamma}\left(t_{0}\right)=0$. Let $\gamma_{0}=\inf \left\{\gamma>a: u_{\gamma}(t)=0\right.$ for a certain $\left.t \in\right] a, \gamma[\}$. Then $u_{\gamma_{0}}(t)>0$ for $\left.t \in\right] a, \gamma_{0}[$ and

$$
\begin{aligned}
& u_{\gamma_{0}}^{(i)}(a)=0 \quad(i=0, \ldots, l-1), \\
& u_{\gamma_{0}}^{(i)}\left(\gamma_{0}\right)=0 \quad(i=0, \ldots, n-l-1),
\end{aligned}
$$

which contradicts our assumption. 
Let $\gamma^{0}=\sup \left\{\gamma>b: u_{\gamma}(t)>0\right.$ for $\left.t \in\right] a, \gamma[\}$. Consider first the case where $\gamma^{0}=+\infty$. There exists the sequence $\left\{\gamma_{k}\right\}_{k=1}^{+\infty}$ such that

$$
\lim _{k \rightarrow+\infty} \gamma_{k}=+\infty, \quad \lim _{k \rightarrow+\infty} u_{\gamma_{k}}(t)=u_{0}(t)
$$

where $u_{0}$ is the solution of Eq. (1). Show that

$$
u_{0}(t)>0 \text { for } t>a \text {. }
$$

It is clear that $u_{0}(t) \geq 0$ for $t>a$. If now $u_{0}\left(t_{*}\right)=0$ for some $t_{*}>a$, then for any $k$ large enough the function $u_{\gamma_{k}}^{\prime}$ will have at least one zero in $] a, \gamma_{k}[$. Taking into account the multiplicities of zeroes of $u_{\gamma_{k}}$ in $a$ and $\gamma_{k}$, it is easy to show that $u_{\gamma_{k}}^{(n-1)}$ has at least two zeroes in $] a, \gamma_{k}\left[\right.$. Hence $u_{\gamma_{k}}^{(n)}$ changes sign in this interval and this is impossible.

Thus inequality (6) is proved. This inequality and the results of [1] imply that there exist $l_{0} \in\{1, \ldots, n\}\left(l-l_{0}\right.$ is even $)$ and $t_{1}>b$ such that

$$
\begin{aligned}
u_{0}^{(i)}(t)>0 & \text { for } t \geq t_{1} \quad\left(i=0, \ldots, l_{0}-1\right), \\
(-1)^{i+l_{0}} u_{0}^{(i)}(t) \geq 0 & \text { for } t \geq t_{1} \quad\left(i=l_{0}, \ldots, n\right) .
\end{aligned}
$$

Clearly,

$$
\begin{array}{lr}
(-1)^{i+l_{0}} u_{0}^{(i)}(t) \geq 0 \quad \text { for } t \geq a & \left(i=l_{0}, \ldots, n\right), \\
(-1)^{i+l_{0}} u_{0}^{(i)}(a)>0 & \left(i=l_{0}, \ldots, n-1\right) .
\end{array}
$$

Hence $l \in\left\{1, \ldots, l_{0}\right\}$.

Suppose that $l<l_{0}$. Then for any $k$ large enough we have $\gamma_{k}>$ $t_{1}, u_{\gamma_{k}}^{(i)}\left(t_{1}\right)>0\left(i=0, \ldots, l_{0}-1\right)$. This means that the function $u_{\gamma_{k}}^{(i)}$ has at least one zero in $] t_{1}, \gamma_{k}[$. Taking into account the multiplicity of zero in $\gamma_{k}$, it is easy to see that $u_{\gamma_{k}}^{(n-1)}$ has at least two zeroes in $] t_{1}, \gamma_{k}\left[\right.$, and $u_{\gamma_{k}}^{(n)}$ changes sign in this interval. But this is impossible. Thus $l=l_{0}$.

As $l=l_{0}$, inequalities (7) and (8) imply

$$
\begin{aligned}
(-1)^{i+l} u_{0}^{(i)}(t) \geq 0 & \text { for } t \geq a \quad(i=l, \ldots, n) \\
u_{0}^{(i)}>0 & \text { for } t>a \quad(i=0, \ldots, l-1) .
\end{aligned}
$$

Let

$$
v(t)=u_{0}^{(l-1)}(t)-\sum_{j=l}^{n-1} \frac{(-1)^{j-l}}{(j-l+1) !}(t-a)^{j-l+1} u_{0}^{(j)}(t) ;
$$

then

$$
v^{\prime}(t)=\frac{(-1)^{n-l}}{(n-l) !}(t-a)^{n-l} u_{0}^{(n)}(t) \geq 0 \text { for } t \geq a
$$


Hence

$$
\begin{aligned}
& u_{0}^{(l-1)}(t) \geq \sum_{j=l}^{n-1} \frac{(-1)^{j-l}(t-a)^{j-l+1} u_{0}^{(j)}(t)}{(j-l+1) !} \geq \\
& \geq \frac{(t-a)(-1)^{n-l}}{(n-l) !} \int_{t}^{+\infty}(s-a)^{n-l-1} p(s) u_{0}(s) d s \text { for } t \geq a .
\end{aligned}
$$

Denote $\rho_{i}(t)=i u_{0}^{(l-i)}(t)-(t-a) u_{0}^{(l-i+1)}(t)$ for $t \geq a(i=0,1, \ldots, l)$. Then $\rho_{i}^{\prime}(t)=\rho_{i-1}(t)$ for $t \geq a(i=1, \ldots, l)$. Since $\rho_{0}(t)=-(t-$ a) $u_{0}^{(l+1)}(t) \geq 0$ for $t \geq a$ and $\rho_{i}(a)=0(i=1, \ldots, l)$, we have $\rho_{i}(t) \geq 0$ for $t \geq a(i=0,1, \ldots, l)$. This implies

$$
u_{0}(t) \geq \frac{(t-a)^{l-1}}{l !} u_{0}^{(l-1)}(t)
$$

From (9) and (10) we obtain

$$
1 \geq \frac{(t-a)}{l !(n-l) !} \int_{t}^{\beta}(s-a)^{n-2}|p(s)| d s \quad \text { for } t \geq a,
$$

which contradicts (5). The case $\gamma^{0}=+\infty$ is thus eliminated.

Now consider the case where $\gamma^{0}<+\infty$. As we have already noted, $\gamma^{0}>b, u_{\gamma^{0}}(t)>0$ for $\left.t \in\right] a, \gamma^{0}[$ and

$$
\begin{aligned}
u_{\gamma^{0}}^{(i)}(a)=0 & (i=0, \ldots, l-1), \\
u_{\gamma^{0}}^{(i)}\left(\gamma^{0}\right)=0 & (i=0, \ldots, n-l-1) .
\end{aligned}
$$

Hence

$$
u_{\gamma^{0}}(t)=\int_{a}^{\gamma^{0}} G(t, s) p(s) u_{\gamma^{0}}(s) d s
$$

where $G$ is the Green's function of the boundary value problem (11) for the equation $u^{(n)}=0$.

Let $\left.t_{0} \in\right] \alpha, \beta[$ be such that

$$
\begin{aligned}
\frac{u_{\gamma^{0}}(t)}{(t-a)^{l-1}\left(\gamma^{0}-t\right)^{n-l-1}} & \geq \frac{u_{\gamma^{0}}\left(t_{0}\right)}{\left(t_{0}-a\right)^{l-1}\left(\gamma^{0}-t_{0}\right)^{n-l-1}} \\
\text { for } t & \in[\alpha, \beta] .
\end{aligned}
$$


Then from the lemma and the inequality (12) it follows that

$$
\begin{aligned}
u_{\gamma^{0}}\left(t_{0}\right) & \geq\left(\gamma^{0}-\beta\right)(\alpha-a) \sum_{i=1}^{n-l} \frac{(-1)^{n-l-i}}{(i-1) !(n-i) !} \times \\
& \times \int_{\alpha}^{\beta} \frac{(s-a)^{n-2}\left(\gamma^{0}-s\right)^{n-2}}{\left(\gamma^{0}-a\right)^{n-1}}|p(s)| d s u_{\gamma^{0}}\left(t_{0}\right)> \\
& >\frac{(b-\beta)(\alpha-a)}{(b-a)^{n-1}} \sum_{i=1}^{n-l} \frac{(-1)^{n-l-i}}{(i-1) !(n-i) !} \times \\
& \times \int_{\alpha}^{\beta}(s-a)^{n-2}(b-s)^{n-2}|p(s)| d s u_{\gamma^{0}}\left(t_{0}\right) .
\end{aligned}
$$

Since $\sum_{i=1}^{n-l} \frac{(-1)^{n-l-i}}{(i-1) !(n-i) !} \geq \frac{1}{l !(n-l) !}$ the inequality (13) contradicts (5).

Denote

$$
\begin{aligned}
& \mu_{n}^{-}=\min \{l !(n-l) !: l \in\{1, \ldots, n-1\}, \quad n-l \text { odd }\} \\
& \mu_{n}^{+}=\min \{l !(n-l) !: l \in\{1, \ldots, n-1\}, \quad n-l \text { even }\}
\end{aligned}
$$

It is clear that

$$
\begin{aligned}
& \mu_{n}^{-}=\left\{\begin{array}{lll}
\left(\frac{n}{2}-1\right) !\left(\frac{n}{2}+1\right) ! & \text { for } & n \equiv 0(\bmod 4), \\
{\left[\left(\frac{n}{2}\right) !\right]^{2}} & \text { for } & n \equiv 2(\bmod 4), \\
\left(\frac{n-1}{2}\right) !\left(\frac{n+1}{2}\right) ! & \text { for } & n \equiv 1(\bmod 2),
\end{array}\right. \\
& \mu_{n}^{+}=\left\{\begin{array}{lll}
{\left[\left(\frac{n}{2}\right) !\right]^{2}} & \text { for } & n \equiv 0(\bmod 4), \\
\left(\frac{n}{2}-1\right) !\left(\frac{n}{2}+1\right) ! & \text { for } & n \equiv 2(\bmod 4), \\
\left(\frac{n-1}{2}\right) !\left(\frac{n+1}{2}\right) ! & \text { for } & n \equiv 1(\bmod 2) .
\end{array}\right.
\end{aligned}
$$

Corollary 1. Let either $n \geq 2, \mu_{n}=\mu_{n}^{-}$, and $p(t) \leq 0$ for $t \in[a, b]$ or $n \geq 3, \mu_{n}=\mu_{n}^{+}$, and $p(t) \geq 0$ for $t \in[a, b]$. Let, moreover, $\left.\alpha, \beta \in\right] a, b[$ exist such that $a<\alpha<\beta<b$ and

$$
\int_{\alpha}^{\beta}|p(t)| d t \geq \mu_{n}\left(\frac{b-a}{(b-\beta)(\alpha-a)}\right)^{n-1}
$$

Then Eq. (1) is conjugate in $[a, b]$.

Note that $\max \{l !(n-l) !: l \in\{1, \ldots, n-1\}\}=(n-1) !$. Thus from Theorem 1 easily follows 
Corollary 2. Let $l \in\{1, \ldots, n-1\}$, let the conditions (4) be fulfilled, and let $\alpha, \beta \in] a, b[$ exist such that $a<\alpha<\beta<b$ and

$$
\int_{\alpha}^{\beta}|p(t)| d t \geq(n-1) !\left(\frac{b-a}{(b-\beta)(\alpha-a)}\right)^{n-1}
$$

Then Eq. (1) is $(l, n-l)$ conjugate in $[a, b]$.

Note that in the inequality $(14)$ the factor $(n-1)$ ! cannot be replaced by $(n-1) !-\varepsilon$ with $\varepsilon \in] 0,1[$. This is shown by the following

Example. Let $\varepsilon \in] 0,1[$ be given beforehand and choose $\alpha \in] a, b[$ and $\beta \in] \alpha, b[$ such that

$$
(n-1) !\left(\frac{\alpha-a}{\beta-a}\right)^{n-1}\left(\frac{b-\beta}{b-a}\right)^{n-1}>(n-1) !-\varepsilon .
$$

Put

$$
\begin{gathered}
v(t)= \begin{cases}t-a & \text { for } t \in[a, \alpha], \\
\frac{\alpha+\beta}{2}-a-\frac{1}{2(\beta-\alpha)}(t-\beta)^{2} & \text { for } t \in] \alpha, \beta[, \\
\frac{\alpha+\beta}{2}-a & \text { for } t \in[\beta, b],\end{cases} \\
u_{0}(t)=\frac{1}{(n-3) !} \int_{a}^{t}(t-s)^{n-3} v(s) d s \quad \text { for } \quad t \in[a, b], n \geq 3, \\
u_{0}(t)=v(t) \quad \text { for } t \in[a, b], n=2,
\end{gathered}
$$

and

$$
p(t)=\frac{v^{\prime \prime}(t)}{u_{0}(t)} \quad \text { for } \quad a<t<b .
$$

Then the function $u_{0}$ is non-decreasing for $t \in[a, \beta]$, and the inequality

$$
u_{0}(t) \leq u_{0}(\beta) \leq \frac{1}{(n-3) !} \int_{a}^{\beta}(\beta-s)^{n-3}(s-a) d s=\frac{(\beta-a)^{n-1}}{(n-1) !}
$$

is valid. Taking into account the inequality (15), we obtain

$$
\begin{gathered}
\int_{\alpha}^{\beta} p(t) d t=\frac{1}{\beta-\alpha} \int_{\alpha}^{\beta} \frac{d t}{u_{0}(t)}>\frac{(n-1) !}{(\beta-a)^{n-1}}> \\
>((n-1) !-\varepsilon)\left(\frac{b-a}{(b-\beta)(\alpha-a)}\right)^{n-1} .
\end{gathered}
$$

On the other hand, in the case considered, Eq. (1) is not conjugate in $[a, b]$ because it has a solution $u_{0}$ satisfying the following conditions:

$$
u_{0}^{(i)}(a)=0(i=0, \ldots, n-2), u_{0}^{(n-1)}(a)=1, u_{0}(t)>0 \text { for } a<t \leq b .
$$


This example shows that in Corollary 2 inequality (14) cannot be replaced by the inequality

$$
\int_{\alpha}^{\beta}|p(t)| d t \geq((n-1) !-\varepsilon)\left(\frac{b-a}{(b-\beta)(\alpha-a)}\right)^{n-1}
$$

no matter how small $\varepsilon>0$ is.

Now consider Eq. (1) on the whole axis $\mathbb{R}$ with $p \in L_{\text {loc }}(\mathbb{R})$. From Corollary 2 easily follows

Corollary 3. Let $l \in\{1, \ldots, n-1\}, \quad p$ is not zero on the set of the positive measure and

$$
n \geq 2+\frac{1+(-1)^{n-l}}{2}, \quad(-1)^{n-l} p(t) \geq 0 \quad \text { for } \quad t \in \mathbb{R} .
$$

Then Eq. (1) is $(l, n-l)$ conjugate in $\mathbb{R}$.

\section{REFERENCES}

1. I. T. Kiguradze, Some singular boundary value problems for ordinary differential equations. (Russian) Tbilisi University Press, Tbilisi, 1975.

2. V. A. Kondratyev, On oscillations of solutions of the equation $y^{(n)}=$ $p(x) y$. (Russian) Trudy Moskov. Mat. Obshch. 10(1961), 419-436.

3. N. L. Korshikova, On zeroes of solutions of linear equations of high orders. (Russian) Differential Equations and Their Applications (Russian), 143-148, Moscow University Press, Moscow, 1984.

4. A. Yu. Levin, Non-oscillation of solutions of the equation $x^{(n)}+$ $p_{1}(t) x^{(n-1)}$

$+\ldots+p_{n}(t) x=0$. (Russian) Uspekhi Mat. Nauk. 24(1969), No. 2, 43-96.

5. A. G. Lomtatidze, On oscillatory properties of solutions of linear differential equations of second order. (Russian) Reports of the seminar of the I. N. Vekua Institute of Applied Mathematics 19(1989), 39-54.

6. T. A. Chanturia, Sturm type theorems of comparison for differential equations of high orders. (Russian) Bull. Acad. Sci. Georgian SSR 99(1980), No. 2, 289-291.

7. - On oscillations of solutions of linear differential equations of high orders. (Russian) Reports of the seminar of the I. N. Vekua Institute of Applied Mathematics 16(1982), 3-72.

8. F. Hartman, Ordinary differential equations. (Russian) Mir, Moscow, 1970; English original, Wiley, New York, 1964.

(Received 1.07.1992) $)^{1}$

\footnotetext{
${ }^{1}$ The original manuscript was prepared for publication by D. Paatashvili
} 